\section{Phospholipase C}

K. J. Lackner ${ }^{1}$ und D. Peetz ${ }^{2}$

${ }^{1}$ Institut für Klinische Chemie und Laboratoriumsmedizin, Universitätsmedizin Mainz, Mainz, Deutschland

${ }^{2}$ Institut für Labormedizin, Helios Klinikum Berlin-Buch, Berlin, Deutschland

\section{Englischer Begriff phospholipase C}

Definition Enzym, das Phospholipide in 1,2-Diacylglyzerol und den jeweils organischen Phosphatrest (Phosphatidylinositol, Phosphocholin etc.) spaltet (s. Abbildung im Stichwort $\checkmark$ Phospholipase A2).
Beschreibung Phospholipase-C-Aktivität findet sich vorwiegend intrazellulär und ist dort meist in Signaltransduktionsprozesse involviert. Insbesondere die Generierung von Inositoltriphosphat und Diacylglyzerol aus Phosphatidylinositol ist von herausragender Bedeutung. Eine Reihe verschiedener Isoenzymfamilien, die mit den griechischen Buchstaben $\beta, \gamma, \delta$ und $\varepsilon$ bezeichnet werden, ist bekannt. Die Regulation dieser Isoenzyme unterscheidet sich z. T. erheblich.

\section{Literatur}

Park JB, Lee CS, Jang JH et al (2012) Phospholipase signalling networks in cancer. Nat Rev Cancer 12:782-792

Yang YR, Follo MY, Cocco L, Suh PG (2013) The physiological roles of primary phospholipase C. Adv Biol Regul 53:232-241 